RELATO DE CASO

\title{
Embolização da reperfusão do aneurisma da artéria poplítea com Onyx ${ }^{\circledast}$
}

\section{Treatment of recurrent popliteal artery aneurysm with Onyx embolization}

Ligia Jackeline lorio Pires' ${ }^{1}$ Lorenza Baptista Diogo ${ }^{2,3}$, Jorge Porto Marassi ${ }^{3}$, Orlando Bonin Silva ${ }^{2,3}$, Lys Nunes dos Santos ${ }^{3,4}$, Monica Rochedo Mayall ${ }^{3,5}$, Marcelo Andrei Sampaio Lacativa ${ }^{6}$, Cristina Ribeiro Riguetti-Pinto ${ }^{6}$

\section{Resumo}

O aneurisma da artéria poplítea é o mais frequente dos aneurismas periféricos verdadeiros. Embora seja assintomático, apresenta complicações isquêmicas por embolia ou trombose. A correção deste aneurisma pode ser cirúrgica convencional, com técnica e prognóstico bem estabelecidos, ou endovascular, técnica inovadora e menos invasiva. A reperfusão do saco aneurismático excluído, apesar de rara, está relacionada à intensa circulação colateral. Relatamos um caso de aneurisma de poplítea, que após dois anos de exclusão apresentou fluxo no saco aneurismático com sintomatologia, sendo tratado por técnica endovacular alternativa.

Palavras-chave: aneurisma; artéria poplítea; embolização terapêutica; dimetil sulfóxido; procedimentos endovasculares.

\begin{abstract}
The popliteal artery aneurysm is the most frequent of the peripheral aneurysms. Although asymptomatic, it can complicate with severe limb ischemia due to embolization or thrombosis. The surgical correction presents well-established techniques and results, while the less invasive endovascular therapy is still evolving. The recurrence is very rare and can be related to collateral artery refilling. We report a recurrent popliteal artery aneurysm after two years of conventional ligation and bypass surgery, in which an alternative endovascular strategy was successfully applied.
\end{abstract}

Keywords: aneurysm; popliteal artery; embolization, therapeutic; dimethyl sulfoxide; endovascular procedures.

\section{Introdução}

Aneurisma é uma dilatação permanente e focal de um vaso, tendo pelo menos $50 \%$ de aumento do diâmetro comparado ao normal esperado ${ }^{1,2} \mathrm{O}$ aneurisma de artéria poplítea corresponde a $12 \%$ do total dos aneurismas, sendo o mais frequente dos periféricos verdadeiros ${ }^{1-3}$. Há maior prevalência no sexo masculino e geralmente está associado a aneurisma em outra localização ${ }^{1-3}$. A grande maioria dos aneurismas de poplítea apresenta etiologia não específica ${ }^{1-3}$. São assintomáticos e a complicação mais frequente é a isquemia por embolia ou trombose $\mathrm{e}^{1-3}$. O tratamento eletivo é preconizado, pois o risco de perda de membro chega a 50\% quando ocorrem complicações ${ }^{4}$.

O tratamento do aneurisma de artéria poplítea pode ser convencional ou endovascular ${ }^{4-7}$. O tratamento cirúrgico convencional por exclusão consiste em ligadura proximal e distal ao aneurisma e confecção de ponte para revascularização. Apesar de ser técnica estabelecida e de prognóstico conhecido, há relatos de reperfusão. O caso relatado é de uma paciente submetida a tratamento convencional por exclusão de aneurisma de artéria poplítea esquerda com reperfusão após dois anos.

Trabalho realizado no Hospital Municipal Salgado Filho (HMSF) - Rio de Janeiro (RJ), Brasil.

${ }^{1}$ Chefe do Serviço de Cirurgia Vascular do HMSF - Rio de Janeiro (RJ), Brasil.

${ }^{2}$ Médicos do Serviço de Cirurgia Vascular do HMSF - Rio de Janeiro (RJ), Brasil.

${ }^{3}$ Alunos do Curso Teórico-prático de Formação em Cirurgia Endovascular (Endocurso 2010) - Rio Janeiro (RJ), Brasil.

${ }^{4}$ Médica do Serviço de Cirurgia Vascular do Grupo Santa Therezinha - Rio de Janeiro (RJ), Brasil.

${ }^{5}$ Médica residente do Hospital Universitário Pedro Ernesto da Universidade do Estado do Rio de Janeiro (UERJ) - Rio de Janeiro (RJ), Brasil.

${ }^{6}$ Coordenadores do Endocurso 2010 - Rio de Janeiro (RJ), Brasil.

Conflito de interesses: nada a declarar.

Submetido em: 11.12.10. Aceito em: 12.06.11.

J Vasc Bras. 2011;10(3):251-255 


\section{Descrição do caso}

Paciente MFAMA, sexo feminino, 52 anos, hipertensa controlada. Foi submetida em 2008 à derivação poplítea esquerda com safena interna reversa e ligadura proximal e distal do aneurisma. Seguiu em acompanhamento ambulatorial, apresentando, dois anos após, recidiva de massa pulsátil em oco poplíteo esquerdo acompanhado de dor intensa.

No estudo de duplex scan, observou-se perviedade da ponte poplítea e reenchimento do saco aneurismático.

No estudo angiográfico diagnóstico, observou-se ligaduras proximal (na artéria poplítea supra-genicular) e distal (na poplítea infra-genicular) presentes e ponte poplítea pérvia, com reabitação do aneurisma por circulação colateral (Figura 1).

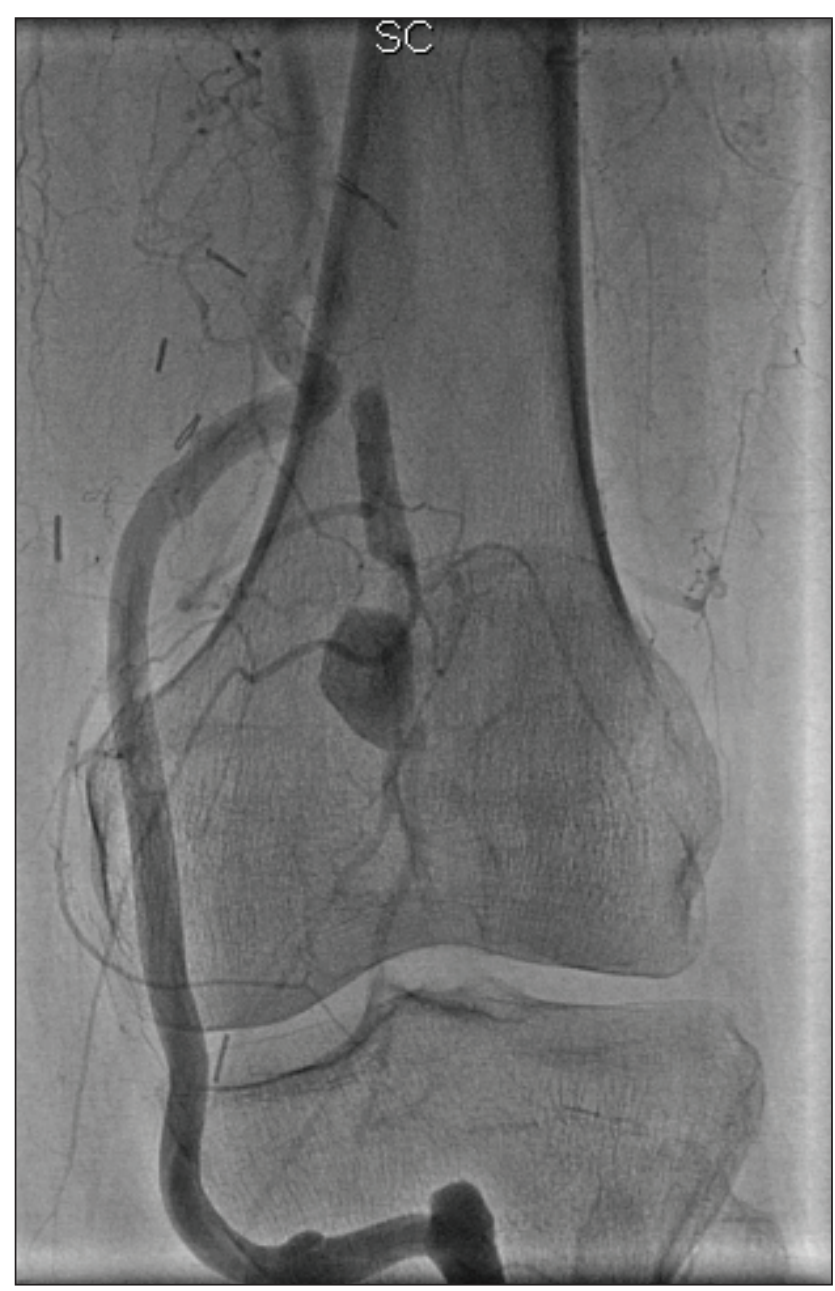

Figura 1. Angiografia diagnóstica evidenciando-se ligadura proximal da artéria poplítea, ponte poplítea pérvia e ligadura distal da poplítea infrapatelar; observa-se também enchimento do aneurisma por múltiplos ramos colaterais.
Optou-se por tratamento endovascular em sala de hemodinâmica, sob raquianestesia. $\mathrm{O}$ acesso utilizado foi a punção da artéria femoral ipsilateral anterógrada, com agulha de punção $18 \mathrm{G}$ e introdução de bainha 5Fr. Realizou-se angiografia do membro inferior esquerdo, identificando-se a origem dos ramos geniculares associados ao saco aneurismático excluído (Figura 2).

Foi efetuado o cateterismo seletivo do ramo genicular superior esquerdo com cateter diagnóstico IM 5F apoiado sobre o fio guia hidrofílico 0,035 pol x $150 \mathrm{~cm}$. Após a injeção de meio de contraste, observamos enchimento do aneurisma de artéria poplítea e refluxo para ramo genicular superior direito e geniculares médios.

Em seguida, realizou-se o cateterismo super seletivo do saco aneurismático (nidus) com microcateter Rebar $10^{\circ}$ (ev3, MA, EUA) sobre fio guia 0,010 pol x $180 \mathrm{~cm}$ (Figura 3). Após a confirmação do posicionamento correto da ponta do microcateter com injeção de contraste não-iônico, observou-se a quantidade de contraste necessária para preenchimento do saco aneurismático e início dos ramos colaterais (Figura 4). O microcateter

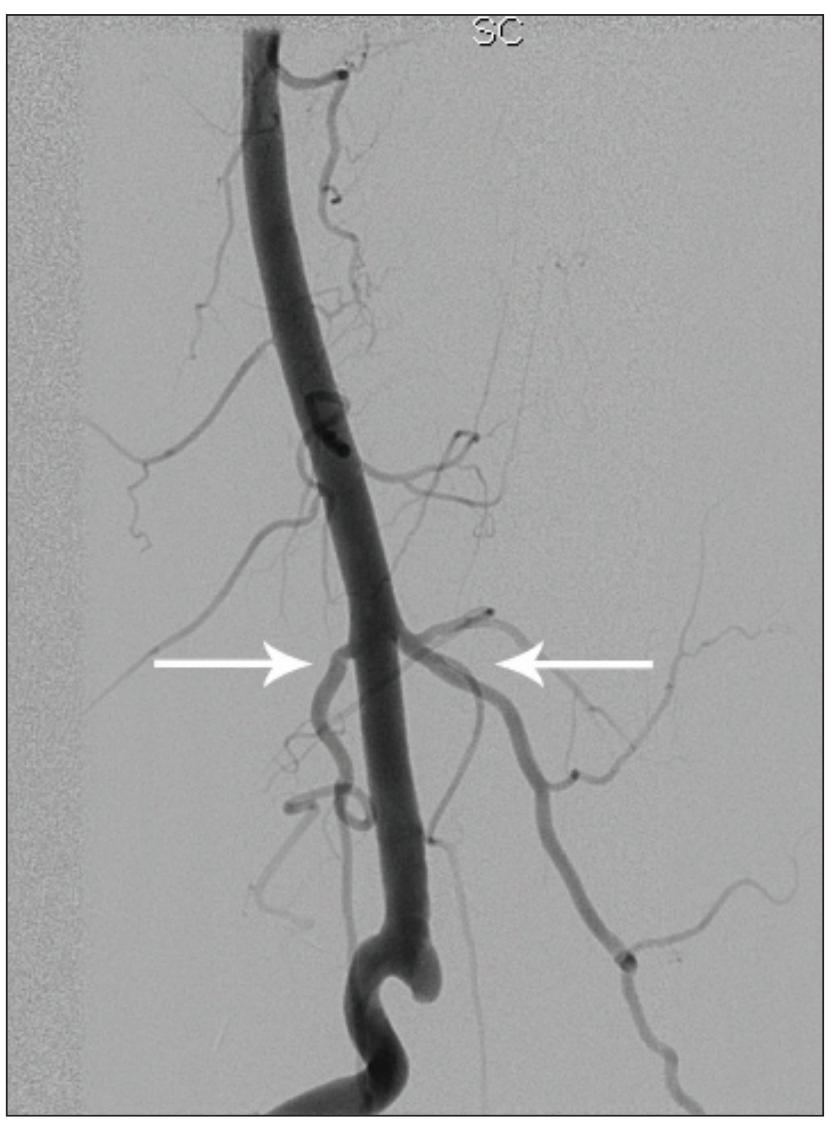

Figura 2. Estudo angiográfico pré-procedimento para identificação da origem dos ramos geniculares superiores indicados pelas setas brancas. 


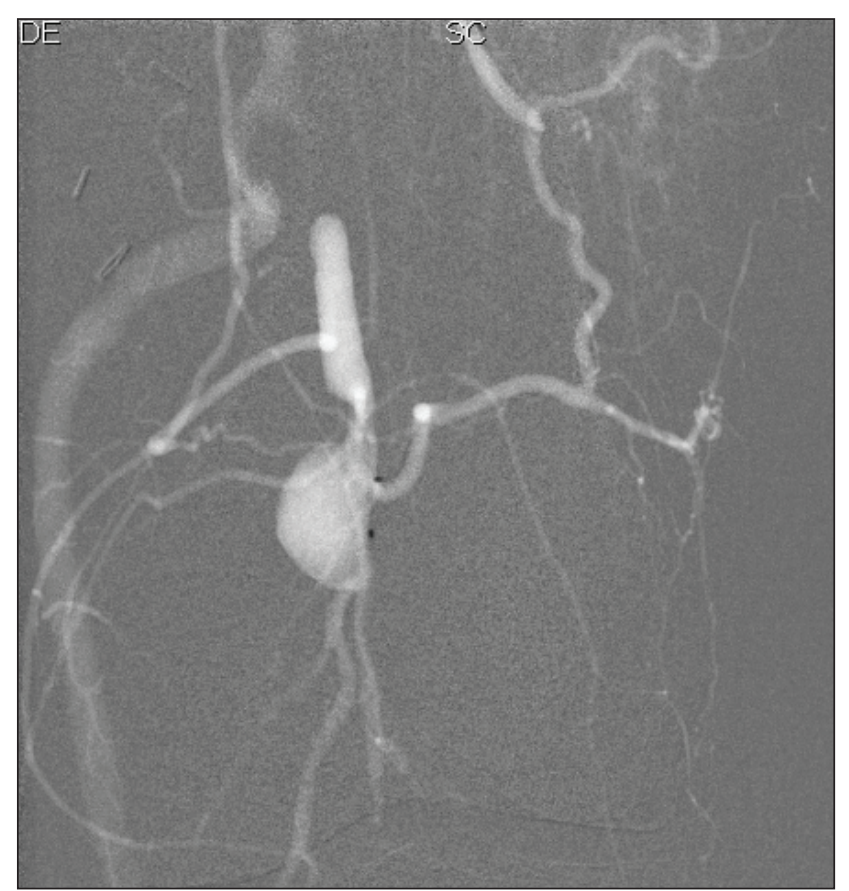

Figura 3. Imagem com roadmapping visibilizando-se microcateter através de genicular superior esquerda, com as duas marcações radiopacas na ponta posicionadas no interior do saco aneurismático.

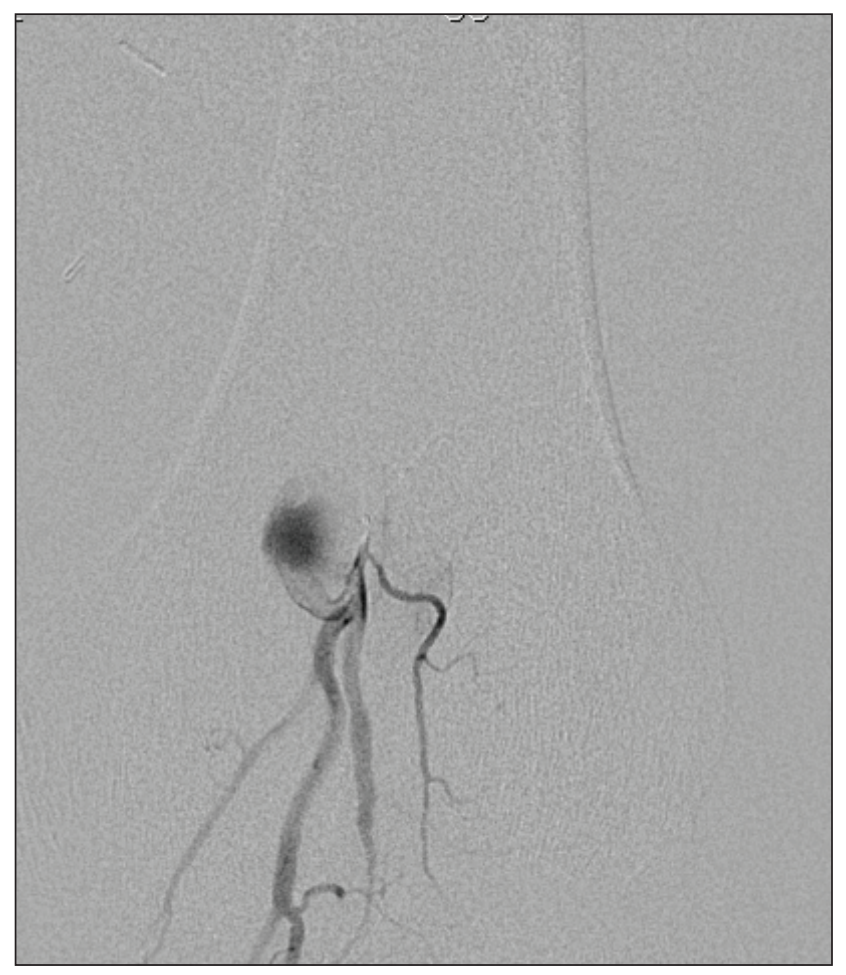

Figura 4. Confirmação do posicionamento correto da ponta do microcateter com injeção de contraste intra saco aneurismático; verificação do volume de contraste necessário para preenchimento do nidus e enchimento da origem dos ramos geniculares. foi preenchido com dimetil sulfóxido (DMSO) e prosseguiu-se com a injeção de 1,5ml de Onyx ${ }^{\circledR}$ (ev3, MA, EUA) (Figura 5).

Na sequência, cateterizou-se seletivamente a artéria genicular superior direita com cateter MP 5 Fr. Introduziu-se um microcateter Rebar $10^{\circledR}$ (ev3, MA, EUA) sobre microfio guia 010 pol, posicionando-se a ponta do mesmo no saco aneurismático, preencheu-se o microcateter com DMSO e injetou-se mais $1,5 \mathrm{ml}$ de Onyx ${ }^{\circledast}$.

Após a injeção de meio de contraste, observou-se bom resultado com fluxo distal preservado, sem enchimento do aneurisma (Figuras 6 e 7).

A paciente recebeu alta no dia seguinte, permanecendo assintomática no acompanhamento ambulatorial. No estudo com mapeamento duplex arterial de controle, um mês após o procedimento, observou-se ausência de fluxo no segmento médio da artéria poplítea e no aneurisma, ponte poplítea pérvia com fluxo trifásico nas artérias distais.

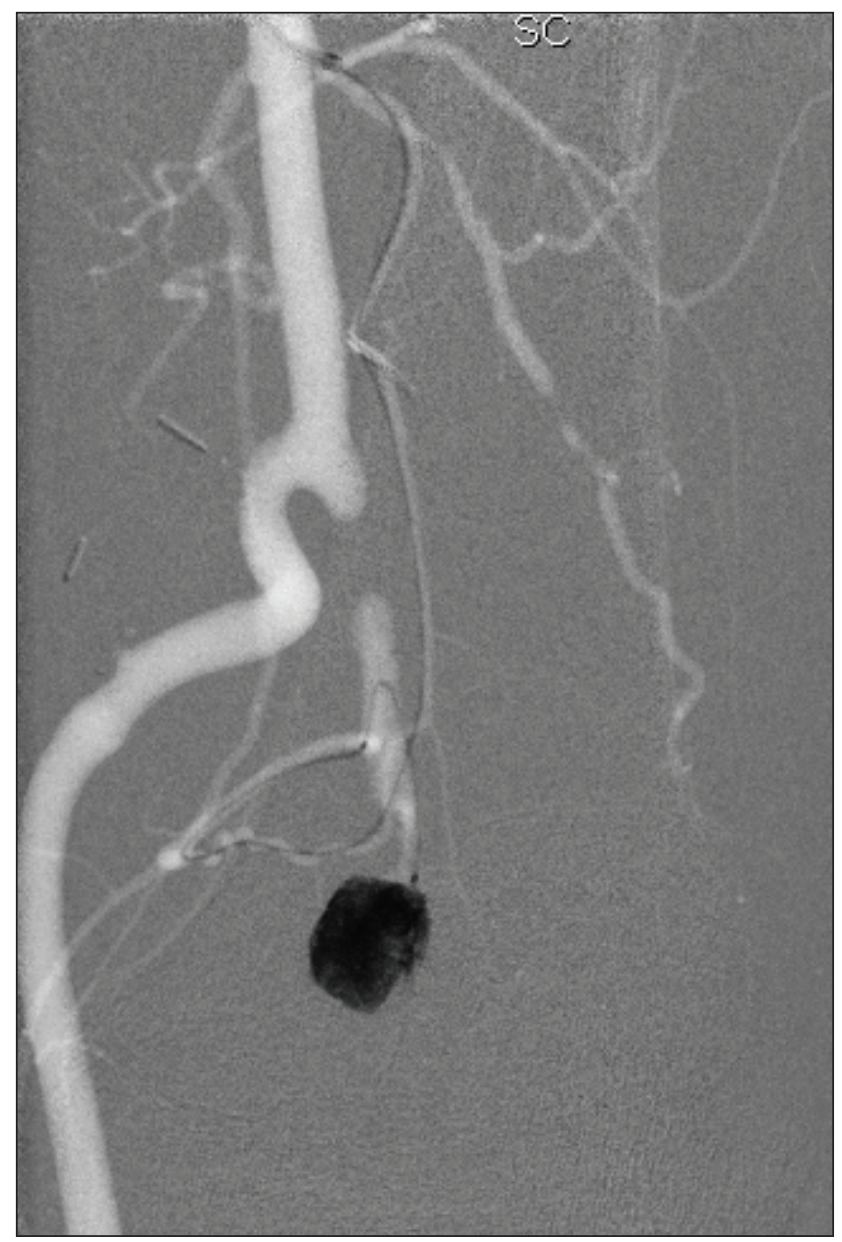

Figura 5. Início da embolização do saco aneurismático com Onyx ${ }^{\circledast}$ em roadmapping 


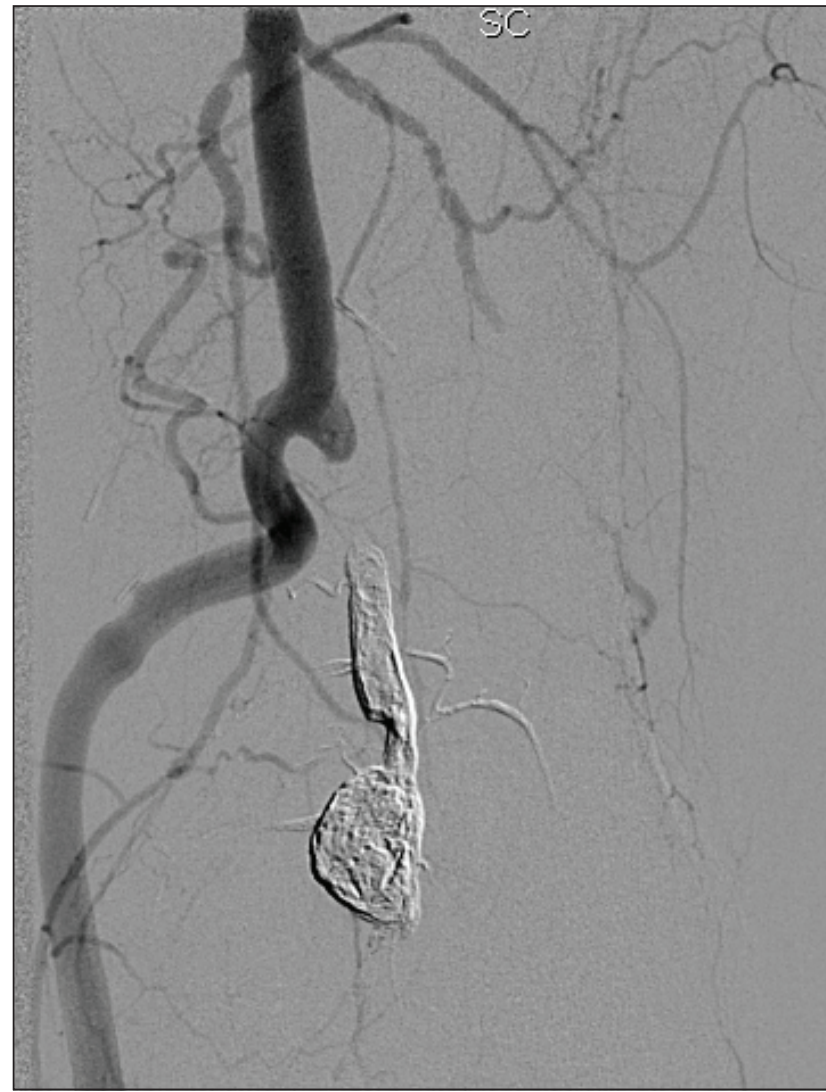

Figura 6. Controle angiográfico em subtração digital, observando-se bom fluxo por ponte poplítea e o material emboligênico radiopaco no saco aneurismático e ramos colaterais.

\section{Discussão}

A cirurgia aberta convencional do aneurisma de artéria poplítea é terapia estabelecida e com resultados satisfatórios a longo prazo $^{1-4}$. A técnica mais utilizada é a exclusão do aneurisma e confecção de derivação fêmoro-poplítea, com índice de sucesso de $90 \%{ }^{4}$. A complicação mais frequente deste tipo de cirurgia é a oclusão da ponte, que pode resultar em sintomas isquêmicos.

A recidiva do aneurisma de poplítea após o tratamento cirúrgico convencional é rara. Sua etiologia está relacionada à presença de fluxo sanguíneo no saco aneurimático pela intensa circulação colateral das geniculares ${ }^{1-4}$. Desta for$\mathrm{ma}$, o aneurisma pode continuar a expandir-se originando sintomas compressivos locais e até evoluir para rotura ${ }^{5}$. O tratamento cirúrgico desta condição pode ser bastante trabalhoso, pelo elevado número de colaterais e dificuldade de identificação dos ramos.

Devido à hemodinâmica semelhante à do vazamento tipo II pós-tratamento endovascular do aneurisma de

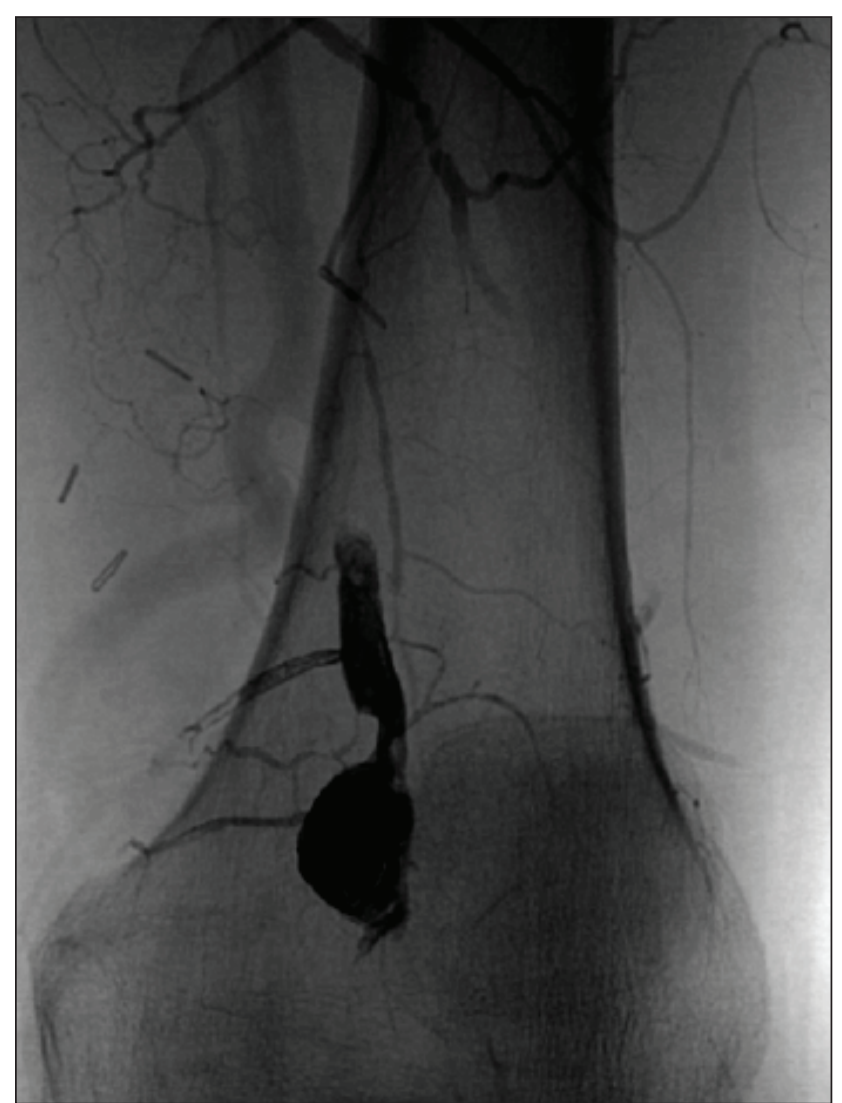

Figura 7. Controle angiográfico em fase tardia em cine, observando-se a substância oclusiva radiopaca no saco aneurismático e na origem dos ramos geniculares colaterais.

aorta abdominal, por ramos colaterais, optamos pela técnica endovascular de embolização ${ }^{8,9}$. Nosso objetivo foi a oclusão do aneurisma e dos ramos geniculares que perfundiam o mesmo.

O agente emboligênico escolhido foi o Onyx ${ }^{\oplus}$, por seu efeito de preenchimento de espaço. Este permite a injeção controlada do centro, ou nidus, com difusão contínua para a periferia, ou início de ramos nutridores. O Onyx ${ }^{\circledR}$ é um material embólico líquido destinado ao uso endovascular; trata-se de um copolímero de etileno vinil álcool, que é dissolvido pelo solvente orgânico DMSO. Quando esta solução entra em contato com meio aquoso, precipita-se e forma um copolímero esponjoso macio, em camadas, com centro geralmente semilíquido ${ }^{8,9}$. O Onyx ${ }^{\circ}$ apresenta maior segurança em relação aos outros agentes líquidos pois permite a injeção em ritmo mais lento e controlado, devido à sua alta viscosidade e maior tempo de polimerização. Outra característica que aumenta a segurança é a radiopacidade acrescida pelo pó de tântalo presente neste agente. Assim, sua visualização é maior em relação às outras substâncias. 
O preenchimento do microcateter com DMSO, imediatamente antes da injeção da solução com Onyx ${ }^{\oplus}$, impede a precipitação do mesmo durante o trajeto. Esta medida permite um comportamento mais previsível, evitando a adesão da ponta do microcateter ao vaso, como pode acontecer com os cianoacrilatos ${ }^{10}$. Quando a mistura entra em contato com o sangue, o DMSO rapidamente se difunde na mistura, causando precipitação e solidificação do polímero in situ, com formação de uma esponja que não adere à parede do vaso ${ }^{11}$.

Apesar de ser indicado para tratamento de endoleaks tipos I e II após implante de endoprótese em aneurisma de aorta abdominal, o Onyx ${ }^{\square}$ só foi aprovado pelo Food and Drug Administration (FDA) para embolização de malformação arteriovenosa (MAV) cerebral ${ }^{8}$. Neste caso em particular, seguimos a estratégia utilizada no tratamento de endoleaks tipo II. Dentre as principais desvantagens na utilização do Onyx ${ }^{\square}$, estão o potencial de vasoespasmo e angionecrose, principalmente associado à injeção rápida do DMSO, que pode ser evitada seguindo-se a recomendação de uso do fabricante de não se ultrapassar a velocidade de injeção de $0,3 \mathrm{ml} / \mathrm{min}$. A isquemia grave de órgãos pode ocorrer, como em qualquer agente emboligênico, caso haja embolização distal inadvertida ${ }^{8}$. Os artefatos ocasionados pelo conteúdo embolizado em estudo de angiotomografia podem dificultar no controle de imagem pós-operatório. Como neste caso a embolização foi em vaso periférico, de fácil abordagem no mapeamento arterial com duplex, o seguimento de imagem provavelmente será satisfatório.

A indicação do tratamento endovascular neste caso foi efetiva, minimizando as chances de recidiva por oclusão direta do saco aneurismático e ramos nutridores, de fácil realização técnica e minimamente invasiva.

\section{Referências}

1. Brito C), Azevedo Junior AC, Schulze GC. Aneurisma Periférico. In: Brito C) (editor). Cirurgia Vascular - Cirurgia Endovascular, Angiologia. Rio de Janeiro: Revinter; 2008. p. 535-540.
2. Buth J, Harris P. Endovascular treatment of aortic aneurysms. In: Cronenwett Il, Gloriczki P, Johnston KW, et al. (editors). Rutherford: Vascular Surgery, 6th ed. Philadelphia: Saunders, 2005; p. 1465.

3. Davidovic LB, Lotina SI, Kostic DM, et al. Popliteal artery aneurysms. World Surg. 1998;22(8):812-7.

4. Medeiros CAF, Gaspar RJ. Correção endovascular do aneurisma da artéria poplítea bilateral. J Vasc Bras. 2006;5(4):303-7.

5. Kauffman P, Puech-Leão P. Tratamento cirúrgico do aneurisma da artéria poplítea: experiência de 32 anos. J Vasc Bras. 2002;1(1):5-14.

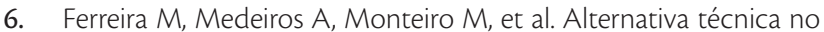
tratamento endovascular dos aneurismas da artéria poplítea. J Vasc Bras. 2008;7(1):44-8.

7. Thomazinho F, Silvestre JMS, Sardinha WF, et al. Tratamento endovascular de aneurisma de artéria poplítea. J Vasc Bras. 2008;7(1):38-43

8. Ling AJ, Pathak R, Garbowski $M$, et al. Treatment of a large type II endoleak via extraperitoneal dissection and embolization of a collateral vessel using ehtylene vinyl alchohol copolymer (Onyx). J Vasc Interv Radiol. 2007;18(5):659-62.

9. Baum RA, Carpenter JP, Golden MA, et al. Treatment of type 2 endoleaks after endovascular repair of abdominal aortic aneurysms: comparison of transarterial and translumbar techniques. J Vasc Surg. 2002(1);35:23-9.

10. Castaneda F, Goodwin SC, Swischuk JL, et al. Treatment of pelvic arteriovenous malformations with ethylene vinyl alcohol copolymer (Onyx). J Vasc Interv Radiol. 2002;13(5):513-6.

11. Muryama Y, Viñuela F, Tateshima S, et al. Endovascular treatment of experimental aneurysms by use of a combination of liquid embolic agents and protective devices. AJNR Am J Neuroradiol. 2000;21(9):1726-35.

Correspondência
Ligia Jackeline lorio Pires
Rua Barão do Bom Retiro, 351 apto 404
CEP 20715-002 - Rio de Janeiro (RJ), Brasil
E-mail: ligiaiorio@oi.com.br
Contribuições dos autores
Concepção e desenho do estudo: LJIP, CRRP, MASL, MRM
Análise e interpretação dos dados: LIIP, CRRP, LDB, JCM
Coleta de dados: LIIP, CRRP, MASL, LNS, OBS
Redação do artigo: LIIP
Revisão crítica do texto: LBD, JCM, OBS, LNS, MASL
Aprovação final do artigo*: LJIP, CRRP, MASL, MRM, LNS,OBS, JCM, LBD
Análise estatística: LIIP, MASL, MRM
Responsabilidade geral pelo estudo: LIIP, CRRP, MASL
*Todos os autores leram e aprovaram a versão final submetida ao J Vasc Bras.

